\title{
Micro-pulse Transscleral Laser Therapy and 'Topical-plus' Anaesthesia: Ideal for Glaucoma Management During COVID-19
}

\author{
Enrico Bernardi1,2 and Marc Töteberg-Harms ${ }^{1,2}$ \\ 1. University Hospital Zurich, Department of Ophthalmology, Zurich, Switzerland; 2. University of Zurich, Medical Faculty, Zurich, Switzerland
}

$\mathrm{M}$ icro-pulse transscleral laser therapy (MP-TLT) is a non-invasive laser procedure for the treatment of glaucoma, and was introduced in 2015. The aim of the procedure is to achieve a reduction in intraocular pressure while minimizing collateral tissue damage. The favourable risk profile of this non-cyclodestructive procedure makes it applicable to a broad spectrum of glaucoma cases, including patients with good central vision, and does not limit its usability to late-stage refractive cases. In 2019, a revised delivery device simplified the procedure, and more recently, a 'topical-plus' anaesthesia protocol has been introduced. The revised delivery system and topical-plus protocol reinforce the utility of MP-TLT as a practical treatment option in an office setting or procedure room, with minimal patient discomfort during and after treatment. Additionally, with minimal follow-up requirements, MP-TLT is ideal for glaucoma management during COVID-19 social restrictions. The following article provides an overview of the use of MP-TLT under topical anaesthesia (topicalplus), the potential role of MP-TLT in the glaucoma treatment algorithm during the COVID-19 pandemic, and the advantages of the revised MicroPulse P3 ${ }^{\oplus}$ probe (IRIDEX Corporation, Mountain View, CA, USA).

\section{Keywords}

Glaucoma, cyclophotocoagulation, MicroPulse, micro-pulse transscleral laser therapy for glaucoma (MP-TLT), MP3, topical-plus anaesthesia

Disclosure: Marc Töteberg-Harms has served as a consultant/advisor for Eyelight and ELT Sight; has received lecture fees from Glaukos Corporation, Heidelberg Engineering, Novartis, Alcon Pharmaceuticals and Théa Pharma; has served as a consultant/advisor for and received lecture fees from, Allergan/AbbVie, MLase

AG and Reichert; has served as a consultant/advisor for and received lecture fees, travel reimbursement and a research grant from IRIDEX; and has served as a consultant/advisor for, and received lecture fees and a research grant from, Santen, Inc. Enrico Bernardi has no financial or non-financial relationships or activities to declare in relation to this article.

Review process: Double-blind peer review.

Compliance with ethics: This study involves a review of the literature and did not involve any studies with human or animal subjects performed by any of the authors.

Authorship: The named authors meet the International Committee of Medical Journal Editors (ICMJE) criteria for authorship of this manuscript, take responsibility for the integrity of the work as a whole, and have given final approval for the version to be published. Access: This article is freely accessible at touchOPHTHALMOLOGY.com. () Touch Medical Media 2021 Received: 6 April 2021

Accepted: 16 August 2021

Published online: 8 December 2021

Citation: touchREVIEWS in Ophthalmology. 2021;15(2):50-4

Corresponding author: Marc Töteberg Harms, University Hospital Zurich, Department of Ophthalmology, Frauenklinikstrasse 24, 8091, Zurich, Switzerland. E: MarcToeteberg@aol.com

Support: No funding was received in

the publication of this article.
Continuous-wave transscleral laser cyclophotocoagulation (CW-TSCPC) is a well-established procedure in the surgical armamentarium of glaucoma management. ${ }^{1}$ It offers a non-incisional option in the treatment of end-stage refractory glaucoma, in which fistulating surgery and maximum tolerated medical therapy have failed to achieve a target intraocular pressure (IOP). ${ }^{2}$

CW-TSCPC is typically delivered using the G-Probe ${ }^{\circledR}$ (IRIDEX Corporation, Mountain View, CA, USA) delivery device, a $600 \mu \mathrm{m}$ quartz fibre-optic probe used with an $810 \mathrm{~nm}$ diode laser (Cyclo G6 ${ }^{\oplus ;}$ IRIDEX Corporation, Mountain View, CA, USA) which is placed over the pars plicata, and delivers discrete high-energy laser spots to coagulate the ciliary body, in order to reduce aqueous humour production, and thus, to reduce IOP.,34 Although CW-TSCPC can be effective, it carries a risk of complications, such as significant intraocular inflammation, pain, conjunctival scarring and burns, cystoid macular oedema, pupillary damage, mydriasis, hypotony and vision loss. ${ }^{5.6}$ These complications potentially limit its use as primary glaucoma surgery.

In contrast to CW-TSCPC, micro-pulse transscleral laser therapy (MP-TLT) for glaucoma incorporates micro-pulse technology to deliver a low-energy therapy. Micro-pulse technology chops a continuous-wave laser beam in an on-and-off cyclical fashion within an envelope of time (Figure 1). The percentage of time that the laser is on within the envelope period is called the 'duty cycle'. It is postulated that during the short on-cycle, heat builds up in the targeted pigmented tissue, while the tissue cools down during the longer off-cycle, avoiding the coagulative threshold. Therefore MP-TLT is a non-cyclodestructive procedure, ${ }^{8}$ and collateral tissue damage is minimized. ${ }^{9}$ Furthermore, in human cadaver eyes, MP-TLT caused less tissue disruption to the ciliary body compared with traditional and low-burn CW-TSCPC treatments. ${ }^{10}$ The exact mechanism of action of MP-TLT is not fully understood; however, investigators have shown evidence that it increases both conventional (trabecular) and alternative (uveoscleral) outflow., ${ }^{8,114}$

In contrast to the G-Probe, the MicroPulse P3 ${ }^{\circledast}$ probe (IRIDEX Corp.) targets the pars plana instead of the pars plicata, and delivers laser energy in a continuous painting, or sweeping, fashion rather than discrete spots. In our practice, we use laser treatment settings of 2,000 mW with a duty cycle of $31.3 \%$, delivering 5-7 sweeps of 11-16 seconds, totalling approximately 80 seconds of treatment time per hemi-circumference. The 3 and 9 o'clock positions are avoided to mitigate chances of pupillary mydriasis by damage to the ciliary nerves. ${ }^{15}$

Outcomes of a randomized, prospective study comparing MP-TLT and CW-TSCPC in eyes with refractory glaucoma, demonstrated lower IOP in the MP-TLT group with similar efficacy to CWTSCPC, which was sustained over 18 months. In addition, MP-TLT was associated with a lower 
Figure 1: Principle of continuous wave and MicroPulse lasers.
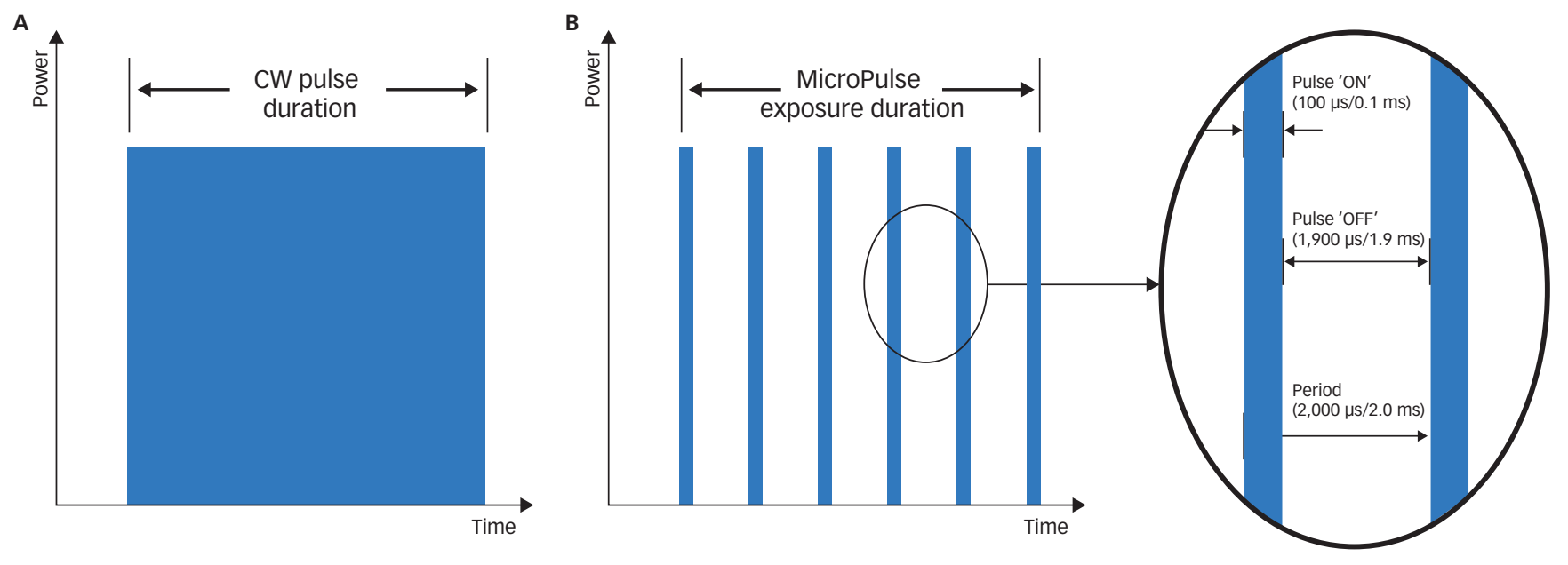

A: With a continuous-wave laser beam, the ciliary body is treated with CW-TSCPC using the G-probe. B: In the micro-pulse treatment mode of the Cyclo G6 ${ }^{\circledR}$ (IRIDEX Corporation Mountain View, CA, USA), the continuous-wave laser is chopped up into brief 'ON' pulses, with longer 'OFF' periods, allowing the tissue to cool down while the laser is off. CW-TSCPC = continuous-wave transscleral laser cyclophotocoagulation.

Figure 2: Glaucoma treatment algorithm at University Hospital Zurich before and during the COVID-19 pandemic

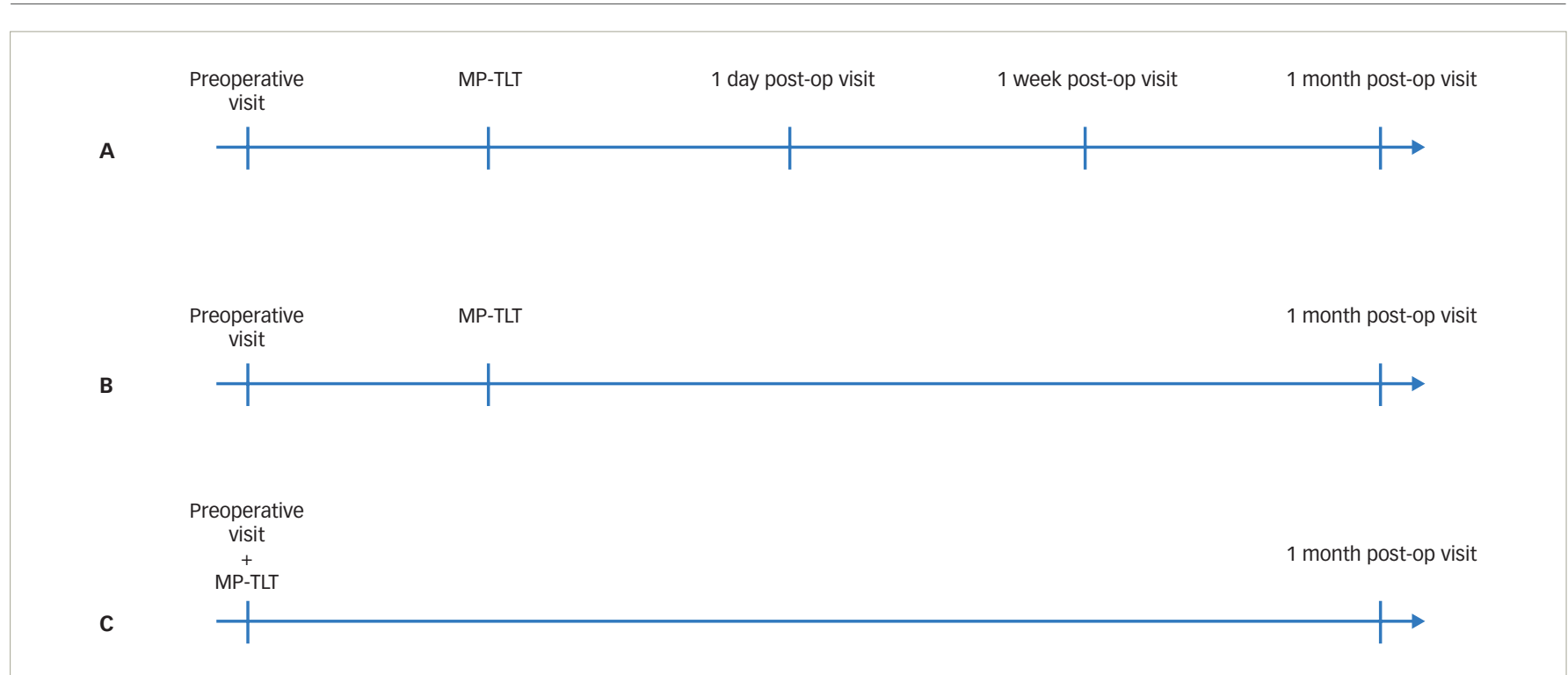

A: Treatment algorithm before COVID-19, where numerous postoperative visits were utilized. B: An example of how viral transmission can be mitigated through limited contact, by reducing postoperative visits to the minimum number clinically indicated after an MP-TLT procedure. C: The final algorithm implemented in our practice, made possible as MP-TLT allows treatment on the same day as the initial consultation.

MP-TLT = micro-pulse transscleral laser therapy.

incidence of vision-threatening complications and a more predictable and consistent effect on IOP reduction. ${ }^{16}$ In our personal experience, MP-TLT is less painful for patients during and after the procedure, and they typically resume their normal activities the following day. Due to the favourable safety profile of MP-TLT, its use is not limited to refractory and end-stage glaucoma, and it can be used in earlier-stage glaucoma prior to incisional therapy, and in patients with good central vision. ${ }^{17}$ The literature cites several studies that demonstrate MP-TLT laser settings and delivery methods that achieve IOP lowering effects in a range of glaucoma severities, with minimal or no serious complications. .14,16-35 $^{-19}$

The aim of this article is to provide an overview of different types of anaesthesia used in association with MP-TLT for the treatment of glaucoma, and how MP-TLT can be added to the surgical treatment algorithm for glaucoma in respect to the COVID-19 pandemic. We provide some insight into our positive experience with MP-TLT during the first wave of the COVID-19 pandemic in Switzerland, and how MP-TLT has enabled us to alter our therapeutic algorithm by reducing the required visits and, thus, decreasing the need for in-person contact and the risk of viral transmission (Figure 2). The algorithm was further improved by the use of the 'topical-plus' as the standard anaesthesia protocol, which allowed for more flexibility in planning laser treatments.

\section{Micro-pulse transscleral laser therapy during the COVID-19 pandemic}

An important aspect to consider during the ongoing COVID-19 pandemic, is the impact of the virus on our clinical practice. SARS-CoV-2 has been found in the tear film; ${ }^{36}$ however, it is unclear whether the virus is present in the tear film due to contamination via rubbing the eye, or if it replicates in the conjunctiva. ${ }^{37}$ As of now, there is no evidence of replicating SARS-COV-2 virus in the aqueous humour or vitreous body. If the virus was present in the intraocular fluids, theoretically, 
aerosolization could be possible during phacoemulsification, and even more so during vitrectomy. ${ }^{38}$

During the first lockdown in Switzerland, and in many other countries, surgeons were allowed to see emergency cases, high-risk patients and patients who would suffer irreversible damage if their treatment was delayed. Thus, the volume of patients at glaucoma clinics and surgical volume decreased significantly during this time. A further implication of the lockdown was the effect on elderly people, who worried about attending the clinic due to the risk of infection, even if consultation or surgery was urgently indicated. In our clinic, we therefore minimized the frequency of patient consultations and changed our treatment algorithm accordingly (Figure 2). Additionally, many colleagues found themselves unable to operate in a surgical theatre due to temporary facility closures.

The benefits of MP-TLT include effectiveness and its favourable safety profile. Another benefit of MP-TLT, which is especially pertinent during the COVID-19 pandemic, is the minimal follow-up consultations associated with this procedure. With the topical-plus anaesthesia protocol (described below), MP-TLT can be performed on the same day as the initial consultation, postoperative medications can be prescribed before the laser procedure, and the first follow-up visit can be scheduled approximately 1 month after treatment. For patients with high IOP refractory to maximum tolerated medical therapy, MP-TLT can be used to postpone incisional surgery. This is especially beneficial during the COVID-19 pandemic, as frequent postoperative consultations are required after incisional surgeries, which can put the patients as risk of a COVID-19 infection. The challenges of available surgical theatre time can be eliminated with MP-TLT, patients' fears may diminish when they know that a non-invasive procedure is planned and patients often appreciate a procedure that allows them rapid recovery with few follow-up visits. While we do not have statistical data, feedback from patients who underwent outpatient MP-TLT treatment, instead of incisional surgery in an inpatient setting during the COVID-19 pandemic, has been positive.

One of the devices used to deliver MP-TLT is the hand-held, fibre-optic MicroPulse P3, which can be used in conjunction with the Cyclo G6. These systems complement each other and have several benefits. The laser is portable and easy to move from the surgical theatre to an examination room, the surface of the laser platform and footswitch can be disinfected, and the MicroPulse P3 probe is non-reusable, thus further minimizing risk of infection. Before the COVID-19 pandemic, MPTLT procedures were conducted in the operating theatre, usually under analgosedation performed by the anaesthesia team. When the first lockdown was initiated in Switzerland in March 2020, we changed to the topical-plus anaesthesia protocol with standby anaesthesia service, and continue to perform nearly all cases under topical-plus anaesthesia, but without standby anaesthesia. Without the requirement for standby anaesthesia, the surgeon can perform MP-TLT wherever the Cyclo G6 laser system is located, whether it is in a small procedure room or simply in an examination room.

\section{Micro-pulse transscleral laser therapy anaesthesia considerations and the 'topical-plus' protocol}

When it comes to the choice of anaesthesia, the following traditional options have been discussed with patients: subconjunctival anaesthesia, sub-Tenon's anaesthesia, parabulbar block or retrobulbar block. Conjunctival bleedings should be avoided due to possible laser energy absorption; therefore, the prior administration of a topical vasoconstrictor (e.g. brimonidine $0.2 \%$ or phenylephrine $5 \%$ ) should be considered. In addition, analgosedation (e.g. propofol plus fentanyl or thiopental plus intravenous fentanyl) can be offered to the patient. Analgosedation with thiopental and fentanyl allows for pain control and sends the patient to sleep for only 2-3 minutes, which is sufficient time for the MP-TLT procedure. Analgosedation eliminates the need for a retrobulbar block and, therefore, removes risk of retrobulbar bleeding or perforating globe injury.

A further option is the topical-plus anaesthesia protocol, which we recently introduced for MP-TLT procedures. It is a combination of topical anaesthesia drops plus lidocaine $2 \%$ non-alcoholic gel, as is sometimes used for cataract surgery. The topical-plus anaesthesia protocol is as follows.

1. Apply topical, unpreserved tetracaine $1 \%$ eye drops $1-3$ times to allow for comfort during insertion of an eye speculum.

2. Insert the eye speculum.

3. Apply lidocaine $2 \%$ gel on the eye surface multiple times over a minimum of 5 minutes. The speculum can be lifted up slightly to give better access for the lidocaine gel to the entire globe, including the subtarsal conjunctiva.

4. Remove the eye speculum.

5. Perform the MP-TLT procedure.

In our experience, this protocol has resulted in minimal complaints of discomfort and pain during and after the treatment, and no pain medication, other than non-steroidal anti-inflammatory drugs on an asneeded basis, has been necessary in the first 24 hours after treatment. Furthermore, in our experience, pain levels during MP-TLT have been so low, that we have not had to discontinue any treatments due to pain. However, this is only based on expert opinion and experience, as no prospective, randomized study has been conducted on this topic. With the topical-plus anaesthesia protocol, we now have a practical option to perform MP-TLT, with or without standby anaesthesia in the operating room, in a small procedure room, or - completely independently from the anaesthesia service - in the office setting (i.e. an ophthalmological examination room)

In our practice, the topical-plus anaesthesia protocol has facilitated the convenient use of MP-TLT in an outpatient setting, where patients can remain comfortable, and are appreciative of the minimal follow-up visits, especially during this time of COVID-19.

\section{The revised MicroPulse P3 ( $\operatorname{Rev} 2$ ) delivery device}

The MicroPulse P3 device has been associated with some challenges, such as uncertainty of probe orientation and placement, maintenance of contact with the globe, stability of the perpendicular orientation on the globe during the sweeping motion and consistent fibre immersion in a fluid interface during the treatment. The revised MicroPulse P3 (Rev 2) delivery device has solved many of these issues. There are several noticeable revisions to the MicroPulse P3 (Rev 2) probe (Figure 3). The tip of the probe was modified from a round convex shape to a concave footplate to match the curvature of the globe, which makes it easier to keep the probe angled correctly and steadily during the sweeping motion. The revised footplate incorporates a recessed fibre (rather than a protruding fibre) and a fluid channel, which maintain a liquid interface to keep the laser energy properly coupled. Additionally, the footplate is reduced in size for easier placement in small eyes, and the 'bunny ears' intuitively guide the user to placement towards the limbus, with the flat side of the footplate directed towards the eyelid. Finally, the stem, which connects the footplate to the probe handle has 
Figure 3: The MicroPulse P3 ${ }^{\circledR}$

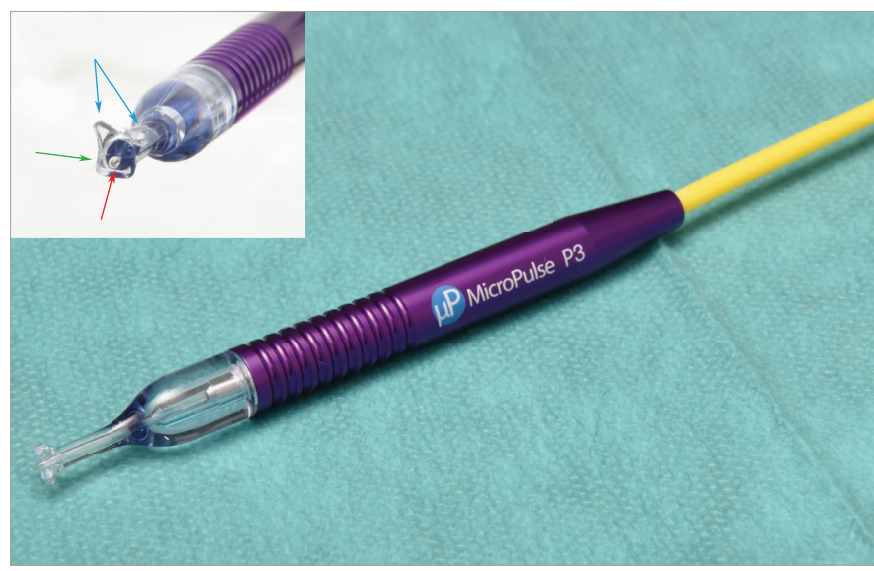

The revised MicroPulse $P 3^{\circledR}$ (IRIDEX Corporation, Mountain View, CA, USA) handpiece has a thin stem, which enables access to the eye without the necessity of a speculum. The 'bunny ears' (blue arrows) at the footplate are oriented towards the limbus. The laser fibre (red arrow) exits the MicroPulse P3 handpiece within a liquid channel (green arrow) at the footplate.

Reproduced with permission from Marc Töteberg-Harms, 2021

been elongated and made thinner to allow better visibility and access to the eye, even if the eyelids are not fully open, or in presence of a smaller palpebral fissure. The stem removes the need for a speculum, thus, enabling better and unobstructed access to the treatment area. Furthermore, forceps are not necessary to rotate and move the eye, preventing subconjunctival haemorrhages.

Treatment with the revised probe includes laser powers of 2,000-2,500 $\mathrm{mW}$, with a duty cycle of $31.3 \%$ delivering 3-5 sweeps of 10-20-second per hemi-circumference or quadrant (Figure 4). During our personal communication with other users of the revised MicroPulse P3 probe, we found that surgeons felt more confident performing the MP-TLT procedure with the revised MicroPulse P3 probe compared with the previous version. Reasons stated for this include increased accessibility to the globe; better visualization of the location of the probe's footplate with its 'bunny ears' in relation to the limbus; and optimal perpendicularity due to the curved design of the footplate, which sits stable on the surface of the globe during the sweeps. Altogether, this also ensures a steeper learning curve for new adapters of this technique.

\section{Post-treatment care}

Immediately after MP-TLT, a steroid eye drop (e.g. prednisolone acetate or dexamethasone sodium phosphate) should be applied to the eye It is common practice for some clinicians to place a subconjunctival depot of dexamethasone and an eye patch. There is, however, currently no consensus on post-treatment care, including the use of topical cycloplegics or antibiotics following MP-TLT, and, therefore, this is left to the surgeon's discretion. Topical steroid drops are usually prescribed for 1-2 weeks (approximately five times daily). Prior glaucoma medications are continued or restarted the morning after the procedure, or reduced depending on the postoperative IOP at the next visit.
Figure 4: Schematic technique of the swipes during micro-pulse laser treatment for glaucoma

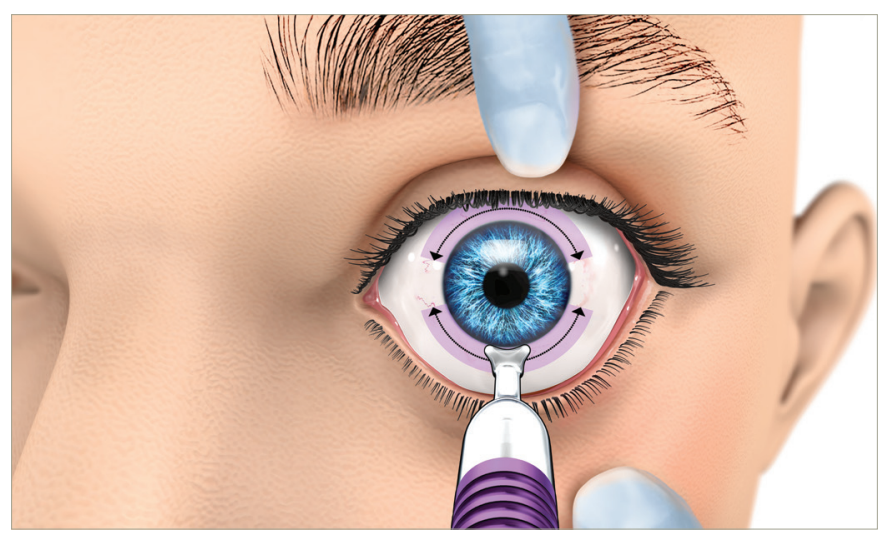

This illustration demonstrates how the laser energy with the MicroPulse P3® (IRIDEX Corporation, Mountain View, CA USA) handpiece is applied to the globe in a sweeping fashion, and not at discrete locations, as it was done with CW-TSCPC CW-TSCPC $=$ continuous-wave transscleral laser cyclophotocoagulation. Reproduced with permission from IRIDEX Corporation, 2021.

From the patient's perspective, the short postoperative recovery time is preferable. Within 24 hours, most of the patients can return to their everyday activities, such as reading or working, with only minimal impact on their lives due to the laser procedure. Fewer follow-up visits, compared with incisional procedures, are required, further decreasing the burden to the patient.

\section{Broad use of micro-pulse transscleral laser therapy}

At the University Hospital Zurich, we have been using MP-TLT since March 2016, as the first centre outside the USA. MP-TLT was initially used for treating severe, end-stage, refractive glaucoma. However, in observing the good safety profile and clinical outcomes, especially regarding visual acuity, ${ }^{17}$ we began incorporating MP-TLT earlier in the management of patients with glaucoma. In our experience, MP-TLT can be offered as an alternative to selective laser trabeculoplasty, prior to incisional surgery in order to postpone incisional surgery or as an alternative to CW-TSCPC. Based on published safety data, MP-TLT can now be considered for use in a broader spectrum of glaucoma cases, including in patients with good central vision. ${ }^{17,39-44}$ One of the benefits of MP-TLT is that, to the best of our knowledge so far, it is not cyclodestructive and, therefore, can be repeated. ${ }^{45}$ However, there is a lack of evidence from randomized controlled trials regarding whether repeated MP-TLT is as effective in lowering IOP compared with primary MP-TLT treatment. The topical-plus anaesthesia protocol allows the surgeon to perform MP-TLT procedures in the operating theatre, in a small procedure room or even in an examination room without standby anaesthesia. MP-TLT has limitations, including the limited IOP lowering effect and the fact that IOP can increase over time following the initial MP-TLT procedure, requiring secondary IOPlowering interventions. $\square$
1. Chen MF, Kim CH, Coleman AL. Cyclodestructive procedures for refractory glaucoma. Cochrane Database Syst Rev. 2019;3:CD012223.

2. Wen JC, Banitt MR. Trans-scleral cyclophotocoagulation curriculum: lecture and skills practice. MedEdPORTAL. 2017;13:10620.

3. Carrillo MM, Trope GE, Chipman ML, Buys YM. Repeated use of transscleral cyclophotocoagulation laser G-probes. J Glaucom 2004;13:51-4.

4. Michelessi M, Bicket AK, Lindsley K. Cyclodestructive procedures for non-refractory glaucoma. Cochrane Database Syst Rev. 2018:4:CD009313.

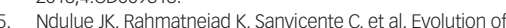

cyclophotocoagulation. J Ophthalmic Vis Res. 2018;13:55-61 6. Winkler NFK, Funk J. Transscleral cyclophotocoagulation as primary surgical intervention in glaucoma. [Article in German Klin Monbl Augenheilkd. 2013;230:353-7.

7. Gaasterland DE. Diode laser cyclophotocoagulation. 2009. Available at: https://glaucomatoday.com/articles/2009-mar/ GT0309_05-php (accessed 17 August 2021).

8. Tan NYQ, Ang M, Chan ASY, et al. Transscleral cyclophotocoagulation and its histological effects on the conjunctiva. Sci Rep. 2019:9:18703.

9. Kuchar S, Moster MR, Reamer CB, Waisbourd M. Treatment outcomes of micropulse transscleral cyclophotocoagulation in advanced glaucoma. Lasers Med Sci. 2016;31:393-6.
10. Maslin JS, Chen PP, Sinard J, et al. Histopathologic changes in cadaver eyes after MicroPulse and continuous wave transsclera cyclophotocoagulation. Can J Ophthalmol. 2020;55:330-5.

11. Barac R, Vuzitas M, Balta F. Choroidal thickness increase after micropulse transscleral cyclophotocoagulation. Rom J Ophthalmol. 2018;62:144-8.

12. Foote BC, Smith JD, Allbaugh RA, Sebbag L. Histologic effects of MicroPulse ${ }^{\mathrm{TM}}$ transscleral cyclophotocoagulation in normal equine eyes. Vet Ophthalmol. 2021;24:59-70.

13. Moussa K, Feinstein M, Pekmezci M, et al. Histologic changes following continuous wave and micropulse transscleral cyclophotocoagulation: a randomized comparative study. TransI Vis Sci Technol. 2020;9:22. 
14. Nguyen AT, Maslin J, Noecker RJ. Early results of micropulse transscleral cyclophotocoagulation for the treatment of glaucoma. Eur J Ophthalmol. 2020;30:700-5.

15. Sanchez FG, Peirano-Bonomi JC, Brossard Barbosa N, et al. Update on micropulse transscleral cyclophotocoagulation. Update on micropulse transs

16. Aquino MCD, Barton K, Tan AMWT, et al. Micropulse versus continuous wave transscleral diode cyclophotocoagulation in refractory glaucoma: a randomized exploratory study. Clin Experiment Ophthalmol. 2015;43:40-6.

17. Varikuti VNV, Shah P, Rai O, et al. Outcomes of micropulse transscleral cyclophotocoagulation in eyes with good central vision. J Glaucoma. 2019;28:901-5

18. de Crom RMPC, Slangen CGMM, Kujovic-Aleksov $\mathrm{S}$, et al. Micropulse trans-scleral cyclophotocoagulation in patients with glaucoma: 1- and 2-year treatment outcomes. I Glaucoma. 2020;29:794-8.

19. Tekeli O, Köse HC. Outcomes of micropulse transscleral cyclophotocoagulation in primary open-angle glaucoma, pseudoexfoliation glaucoma, and secondary glaucoma. pseudoexfoliation glaucoma, and secon
Eur J Ophthalmol. 2021;31:1113-21.

20. Preda MA, Karancsi OL, Munteanu M, Stanca HT. Clinical outcomes of micropulse transscleral cyclophotocoagulation in refractory glaucoma-18 months follow-up. Lasers Med Sci. 2020;35:1487-91

21. Al Habash A, AlAhmadi AS. Outcome of MicroPulse ${ }^{\oplus}$ transscleral photocoagulation in different types of glaucoma. Clin Ophthalmol. 2019;13:2353-60.

22. Souissi S, Baudouin C, Labbé A, Hamard P. Micropulse transscleral cyclophotocoagulation using a standard protocol in patients with refractory glaucoma naive of cyclodestruction. Eur J Ophthalmol. 2021;31:112-9.

23. Jammal AA, Costa DC, Vasconcellos JPC, Costa VP. Prospective evaluation of micropulse transscleral diode cyclophotocoagulation in refractory glaucoma: 1 year results. Arq Bras Oftalmol. 2019:82:381-8.

24. Zaarour $\mathrm{K}$, Abdelmassih $\mathrm{Y}$, Arej N, et al. Outcomes of micropulse transscleral cyclophotocoagulation in uncontrolled glaucoma patients. J Glaucoma. 2019;28:270-5.

25. Sarrafpour S, Saleh D, Ayoub S, Radcliffe NM. Micropulse transscleral cyclophotocoagulation: a look at long-term effectiveness and outcomes. Ophthalmol Glaucoma. 2019;2:167-71.

26. Garcia GA, Nguyen $C$ V , Yelenskiy $A$, et al. Micropulse transscler diode laser cyclophotocoagulation in refractory glaucoma: shortterm efficacy, safety, and impact of surgical history on outcomes. Ophthalmol Glaucoma. 2019;2:402-12.

27. Sanchez FG, Lerner F, Sampaolesi J, et al. Efficacy and safety of Micropulse ${ }^{\oplus}$ transscleral cyclophotocoagulation in glaucoma. Arch Soc Esp Oftalmol (Engl Ed). 2018;93:573-9.

28. Williams AL, Moster MR, Rahmatnejad K, et al. Clinical efficacy and safety profile of micropulse transscleral cyclophotocoagulation in refractory glaucoma. J Glaucoma. 2018;27:445-9.

29. Emanuel ME, Grover DS, Fellman RL, et al. Micropulse cyclophotocoagulation: initial results in refractory glaucoma. J Glaucoma. 2017;26:726-9.

30. Tan AM, Chockalingam M, Aquino MC, et al. Micropulse transscleral diode laser cyclophotocoagulation in the treatment
to of refractory glaucoma. Clin Exp Ophthalmol. 2010;38:266-72.

31. Fili S, Kontopoulou K, Vastardis I, et al. Transscleral cyclophotocoagulation with MicroPulse ${ }^{\circledast}$ laser versus Ahmed valve implantation in patients with advanced primary open-angle glaucoma. Int Ophthalmol. 2021;41:1271-82.

32. Lim JYE, Cecilia AM, Lim KAD, et al. Clinical efficacy and safety outcomes of micropulse trans-scleral diode cyclophotocoagulation in patients with advanced glaucoma. J Glaucoma. 2021;30:257-65.

33. Scheres LMJ, Kujovic-Aleksov S, Ramdas WD, et al. XEN® Gel Stent compared to PRESERFLOTM Microshunt implantation for primary open-angle glaucoma: two-year results. Acta Ophthalmol. 2020;99:e433-40.

34. Radhakrishnan S, Wan J, Tran B, et al. Micropulse cyclophotocoagulation: a multicenter study of efficacy, safety, and factors associated with increased risk of complications. J Glaucoma. 2020;29:1126-31.
35. Kaba Q, Somani S, Tam E, Yuen D. The effectiveness and safety of micropulse cyclophotocoagulation in the treatment of ocular hypertension and glaucoma. Ophthalmol Glaucom 2020;3:181-9.

36. Napoli PE, Nioi M, d'Aloja E, Fossarello M. The ocular surface and the coronavirus disease 2019: does a dual "ocular route" exist? Clin Med. 2020;9:1269

37. Xia J, Tong J, Liu M, et al. Evaluation of coronavirus in tears and conjunctival secretions of patients with SARS-COV-2 infection. J Med Virol. 2020;92:589-94.

38. Chodosh J, Holland GN, Yeh S. Special considerations for ophthalmic surgery during the COVID-19 pandemic. 2020. Available at: www.aao.org/headline/special-considerationsophthalmic-surgery-during-c (accessed 18 August 2021).

39. Waibel S, Herber R, Pillunat LE, Pillunat KR. One-year follow-up of pars plicata versus pars plana application of transscleral micropulse cyclophotocoagulation. J Glaucoma 2021:30:340-6.

40. Maestri F, Legrand M, Da Cunha E, et al. Micropulsed diode laser transscleral cyclophotocoagulation: an effective technique whose role remains to be defined. [Article in French] $J \mathrm{Fr}$ whose role remains to be
Ophtalmol. 2021:44:350-7.

41. Souissi S, Le Mer Y, Metge F, et al. An update on continuous-wave cyclophotocoagulation (CW-CPC) and micropulse transscleral laser treatment (MP-TLT) for adult and paediatric refractory glaucoma. Acta Ophthalmol. 2021;99:e621-53.

42. Ariga M, Nivean, Nivean PD, et al. Micropulse trans-scleral diode laser cyclophotocoagulation in refractory glaucoma: an initial experience in Indian eyes. Int Ophthalmol. 2021;41:2639-45.

43. Lim JYE, Cecilia AM, Katherine LWX, et al. Efficacy and safety of repeated micropulse trans-scleral diode cyclophotocoagulation in advanced glaucoma. J Glaucoma. 2021;30:566-74.

44. Dastiridou Al, Katsanos A, Denis P, et al. Cyclodestructive procedures in glaucoma: a review of current and emerging options. Adv Ther. 2018:35:2103-27.

45. Lim EJY, Cecilia AM, Lim DKA, et al. Clinical efficacy and safety outcomes of micropulse transscleral diode cyclophotocoagulation in patients with advanced glaucoma J Glaucoma. 2021;30:257-65. 